\title{
Are you addicted to
}
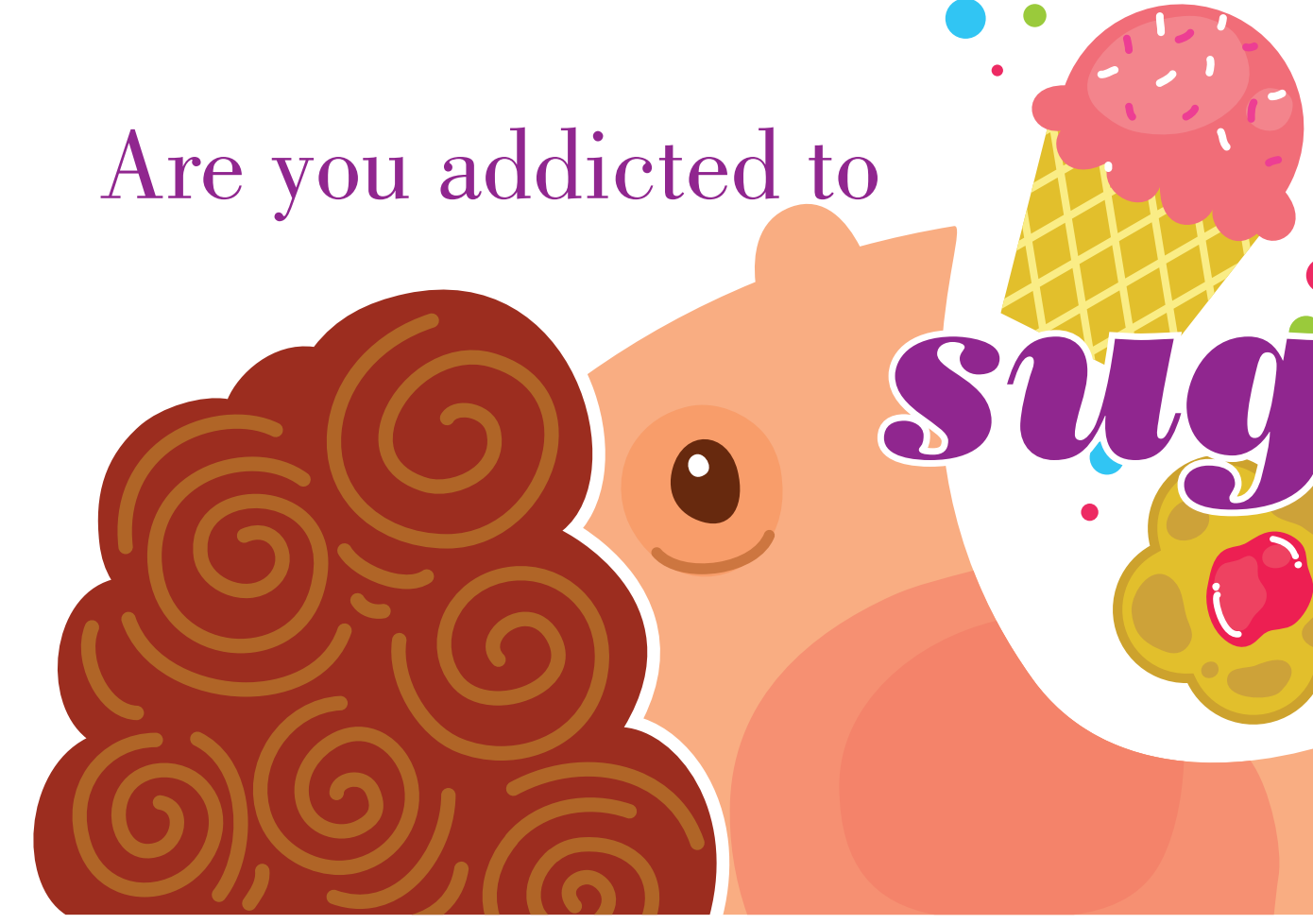

\section{As a dental professional you often advise patients to cut down their sugar intake, but are your own sugar cravings under control? asks Laura Thomas?}

\section{Practising what you preach}

You know that your patients' sugar habits end up costing them greatly in terms of dental treatment and you're regularly dealing out low sugar lifestyle advice to help them. However, sometimes you find yourself craving sugar and succumbing to a few more chocolates than you'd like. Keen to live by your messages, what can you do to get more control over your own sugar cravings and protect your own health from the white stuff?

\section{Other impacts of sugar}

In addition to attacking teeth and enamel, an excess of sugar has all sorts of other detrimental impacts on your body.

Refined sugar, whether in its raw form or

' Laura Thomas is a certified health coach and the founder of Happy Sugar Habits, an organisation working to educate and support individuals to reduce their sugar consumption, and feel a sense of control over their sweet cravings. Laura runs a successful Mentor Me Off Sugar detox programme and speaks nationally about sugar, emotional eating and the practicalities of a low sugar lifestyle. Laura was the former the presenter of The Sugar Diaries radio show for UK Health Radio, has written for The Telegraph and the Daily Mail and has recently been featured in Elle and Grazia magazines. Find out more at www. happysugarhabits.com in a processed food like a tomato sauce, is made up of $50 \%$ glucose and $50 \%$ fructose. You need to know that fructose is metabolised very differently to glucose - it's processed exclusively by the liver. Consuming an excess of fructose (sugar) puts serious strain on this vital organ which can lead to a number of ailments including non-alcoholic fatty liver disease and elevated levels of uric acid (contributing to gout). It's also worth noting that excess fructose consumption over a sustained and long period of time can increase the risk of the serious chronic diseases (eg diabetes) that are plaguing the Western world today.

Sugar also plays havoc on your blood sugar management, impacting on your sleep quality, concentration and energy levels. It weakens your immune system and speeds up the ageing process.

Finally, sugar is addictive. Over time, a gradual increase in consumption can start to negatively affect your behaviour. This can result in you needing more sweet food to satisfy your sweet tooth.

Reminding yourself of these reasons can help motivate you to take consistent actions to reduce the sugar in your diet.

So what can you do to shift your taste buds away from sweet?

\section{Stop drinking sugar}

It's likely that because you know about the detrimental impacts of sugary soft drinks on teeth and enamel, you aren't chucking back half a dozen fizzy drinks during the day. However, it's worth being aware of the more covert sources which may be exposing your palate frequently to the sweet taste that drives cravings.

The sweetener in your tea, the smoothie drink on the go or that tonic water in your gin tipple are all still sweet drink sources that you may not realise have become somewhat habitual in your routine or lifestyle. Seek to identify those last few places where you are drinking sugar and work to eliminate sugar in all liquid forms. Adapt to unsweetened tea or coffee; use fresh lemon or lime to flavour water [editor's note - but use a straw to protect your tooth enamel!] and opt for the whole fibre rich fruit instead of a sugar condensed smoothie.

\section{Lower sugar substitutes of your favourite foods}

Quite often there can be a big difference in sugar content between brands of the same food. Spending a little time researching and finding the ones that don't have as much added sugar shaves off a few grams that can make a difference if you're eating these products daily. Muesli, sauces and dressings are all good items to do this with. By doing this you're also becoming aware of the great lower sugar products that you can easily recommend to your patients. 


\section{Being}

\section{mindful of}

\section{total sugar}

(especially fructose)

As mentioned earlier,

fructose is the more

dangerous part of sugar.

Although fruits and natural sources of sugar have nutritional benefit and are healthy in many respects, they are still sweet and you're likely to still experience powerful cravings if you're consuming them excessively.

To keep cravings at bay, be mindful of the total amount of sweet food in your diet (including fruit), the quantities you're consuming and how often you're eating it. Be particularly wary of dried fruit products and fruit purees that tend to be very concentrated sources of sugar. If something says 'refined sugar free' always check that it's not just been jam packed full of 'natural' dried fruit sugar.

\section{Avoid hidden sugar}

It goes without saying: avoid sugar that you don't know you're eating. Double check sauces, dressings, cereals, soups etc. Look to make your own where you can and cut back on processed foods as much as possible.

\section{Consider your use of sugar}

It's very common for people to use sugar as a stress coping mechanism. It's accessible, cheap, quick and easy. Take note if you're consuming sugar in response to emotional hunger. Seek to build in other coping mechanisms that don't involve sweet food eg some yoga or a run, something relaxing like a walk or a simple breathing exercise.

\section{Embrace the savoury foods you love}

To avoid the doom and gloom feeling of eating less sugar, embrace your favourite savoury foods in all their forms. Try new combinations of them, be more experimental with foods and try to enjoy the process of finding savoury alternatives that you really get excited about.

\section{Work through social challenges}

Often it's the social side of sugar that can be particularly challenging: the birthdays, weddings and numerous annual occasions that are closely associated with excessively sugary food. Seek to understand if you're eating sugar because

RECIPE: Savoury olive \& almond flapjacks
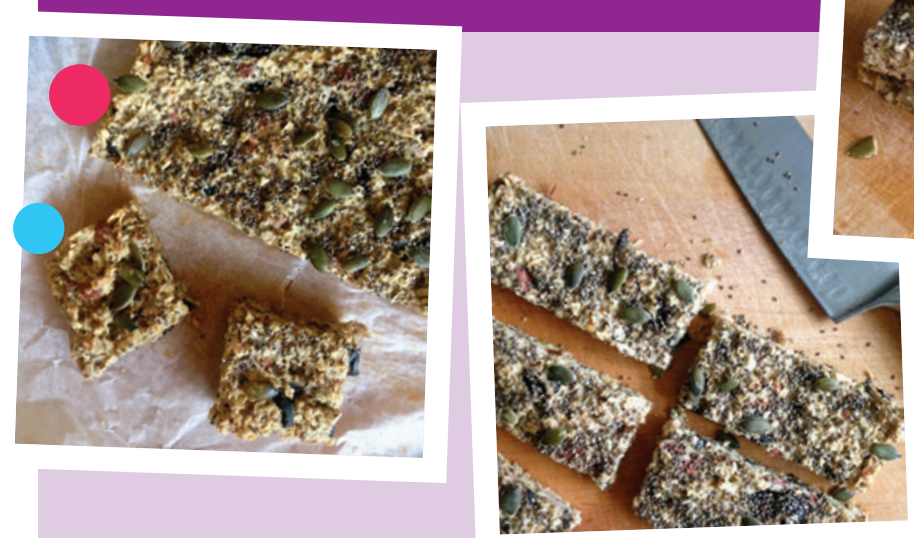

These have all the satisfaction of a flapjack but with a savoury twist and no sugar in sight. Great as a quick breakfast bar or as a substantial snack. The pumpkin and chia seed topping is optional to simply boost the nutritional count and make them look pretty!

Makes 8 large bars or 16 smaller bites

Ingredients

$200 \mathrm{~g}$ rolled oats (2 cups)

$300 \mathrm{ml}$ milk ( $1 \frac{112 \mathrm{cups})}{}$

$75 \mathrm{~g}$ almonds (1/2 cup)

$20 \mathrm{~g}$ parmesan cheese ( $1 / 3$ cup, grated)

$60 \mathrm{~g}$ black pitted olives ( $1 / 2$ cup)

$1 / 2$ tsp salt

2 tbsp dried oregano

2 tbsp ground flaxseed

1 tsp cumin seeds

1 tsp chilli flakes

1 tbsp chia seeds (optional)

1 tbsp pumpkin seeds (optional)

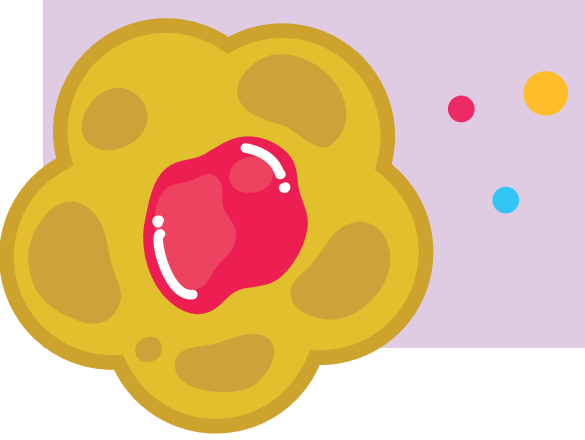

everyone else is, or if it's closely tied to the joy you feel at these events and celebrations. Building awareness of your sugar habits in social situations is the first best step to adjusting them.

For more tips, recipes and a free low sugar snack guide to get control over sugar cravings visit and sign up for updates at www. happysugarhabits.com.

\section{Method}

- Preheat the oven to $180 \mathrm{C}$ and line an 8-inch square tin with parchment paper

- In a medium bowl, mix the oats, salt, herbs and spices together with the ground flaxseed

- Add the milk and leave it to soak for 10 minutes whilst you prepare the rest

- Roughly chop the olives, almonds and grate the parmesan cheese

- Add these ingredients to the mixture stir well

- Pour the mixture into the tin and spread evenly

- Sprinkle over the chia seeds and pumpkin seeds

- Bake for 45 minutes, remove and let cool

- Slice into bars or squares

- Keep refrigerated in an airtight container for up to 3 days
Have you had a go at this recipe? Let us know how your flapjacks turned out and send a photo of the results to bdjteam@nature.com or upload it on the BDJ Team Facebook page www. facebook.com/bdjteam. 